\title{
The effect of in-hospital physiotherapy on handgrip strength and physical activity levels after cardiac valve surgery: a randomized controlled trial
}

\author{
Jinxiu Chen, Tianfang Zhang, Wangxiao Bao, Guiying Zhao, Zuobing Chen \\ Department of Rehabilitation Medicine, The First Affiliated Hospital, Zhejiang University School of Medicine, Hangzhou, China \\ Contributions: (I) Conception and design: J Chen, Z Chen; (II) Administrative support: Z Chen; (III) Provision of study materials or patients: J Chen, \\ T Zhang, G Zhao; (IV) Collection and assembly of data: J Chen, T Zhang, G Zhao; (V) Data analysis and interpretation: J Chen, W Bao; (VI) \\ Manuscript writing: All authors; (VII) Final approval of manuscript: All authors. \\ Correspondence to: Zuobing Chen. The First Affiliated Hospital, Zhejiang University School of Medicine, Hangzhou 310000, China. \\ Email: czb1971@zju.edu.cn.
}

\begin{abstract}
Background: Patients who undergo cardiac valve surgery undertake routine physical therapy program. Despite its routine use, its influence on physical activity level post- surgery has not been illustrated. This study was to investigate whether 5 days of in-hospital physiotherapy could improve physical activity levels after cardiac valve surgery.

Methods: The study is a single-blind randomized controlled trial which performed in Cardiothoracic Surgery Department. Patients who underwent cardiac valve surgery $(n=34)$ for confirmed cardiac valve disorders were assessed during hospitalization. The intervention group received a daily post-operative physiotherapy intervention, consisting of individualized mobilization, breathing exercises, ambulation with or without a walking aid. There was no physiotherapy treatment in the control group. Measurements: physical activity was assessed with the handgrip strength test and the timed up and go test.

Results: The treatment group showed significantly greater handgrip strength [20.58 (7.17) vs. $12.96(4.65) \mathrm{kg}]$ and less time on the timed up and go test [5.92 (2.91) vs. $6.53(1.60) \mathrm{s}]$ compared to the control group on the 5 th post-operative day. Whilst there was no significant difference on the timed up and go test between the 2 groups, handgrip strength on the 5 th post-operative day was significantly different between the 2 groups.

Conclusions: Patients who received physiotherapy during hospitalization showed increased levels of handgrip strength and physical activity on the 5th day after cardiac valve surgery compared to the control group. The clinical value of increased levels of physical activity after in-hospital physiotherapy following cardiac valve surgery requires further investigation.
\end{abstract}

Koywords: Physiotherapy; physical therapy; cardiac valve surgery; randomized controlled trail; handgrip strength; physical activity

Submitted Sep 29, 2020. Accepted for publication Jan 26, 2021.

doi: 10.21037/apm-20-2259

View this article at: http://dx.doi.org/10.21037/apm-20-2259

\section{Introduction}

With increasing prevalence due to an aging group, and in despite of advances in treatment techniques, heart valve disorders comprise nearly one-third of heart diseases (1). At present, degenerative heart valve disease is the most common form of the disease, though rheumatic heart disease, which affects the valves, was previously more common (2). Heart valve disease is either right-sided (tricuspid and pulmonary valves), left-sided (mitral and aortic valve disease), or a combination of the two (2). Initially, heart valve disease is 
usually asymptomatic, but when they present, symptoms include dyspnea, fatigue, and decreased physical capacity. Symptomatic heart valve disease significantly impacts physical function and quality of life, and is also associated with morbidity and mortality (1). When severe symptoms present, the treatment of choice is valve surgery to repair impaired valves, or valve replacement (2).

Before valve surgery, inactivity as a result of dyspnea and physical inability is common. After surgery, people are often immobilized. As cardiac surgery is an unusual and very stressful life event (3), and quality of life may be influenced (4), some patients can experience mental health problems, including depression and anxiety (5). A Cochrane review (6) indicated that people who underwent coronary artery bypass graft surgery benefited from psychological treatments (5). Little is known about the impact of psychological interventions in patients postoperatively.

Cardiovascular diseases are main causes of hospital admission and mortality. Cardiac surgery is differentiated from other sorts of treatment as a result of developments in techniques and materials which have led to a safer procedures and lower risks during the perioperative period. Despite these improvements, complications are still frequent during the post-operative period, and are a determinant of functional recovery and length of hospital stay (7). In one study of 204 patients with cardiovascular diseases, 58\% of them had post-operative complications, mainly in the respiratory system (31\%), cardiovascular system (15.8\%), and central nervous system (13.9\%) (7). The most significant contributor to post-operative complications is prolonged bed rest. Post-operative bed rest can contribute to multiple organ system dysfunction. Immobilization impairs oxygen transport ability, increases the risk of developing lower extremity deep vein thrombosis and even pulmonary thromboembolism, and causes decrease in muscle mass and muscle strength. In spite of these negative effects, bedding is prescribed after surgery. As for patients who had cardiac surgery, activity limitation has been presented to diminish cardiac overload (8). By comparison, recently, some studies have focused on the benefit of early mobilization for improving oxygen transport ability and functional rehabilitation, reducing complications post-surgery, and shortening the duration of hospitalization (8-11).

Early mobilization after surgery has many benefits, including increasing ventilation, muscle strength, and functional ability (9). A systematic review that analyzed 15 randomized clinical trials on early mobilization in intensive care units (ICUs) showed that early mobilization is practical, safe, and has an active effect on functional performance (12). And breathing exercises separately are insufficient to prevent post-operative complications. Therefore, this study aimed to investigate whether inhospital physiotherapy during the early post-operative period could improve physical activity levels after cardiac valve surgery.

We present the following article in accordance with the CONSORT reporting checklist (available at http://dx.doi. org/10.21037/apm-20-2259).

\section{Methods}

\section{Study participants}

This was a single-blind randomized controlled trial conducted between 2018 to 2019 in the First Affiliated Hospital, Medical College of Zhejiang University. In this study, 34 patients who were undergoing cardiac valve surgery were randomly divided into 2 groups, except for 1 patient who refused surgery: an intervention group $(n=17)$ and a control group $(n=16)$, according to the computergenerated randomization list. Patients who did not have a history of severe spinal cord injury, stroke, or any other diseases that could cause lower extremity impairment were eligible to participate in the study. The exclusion criteria were unstable hemodynamics, unconsciousness, and patients who are in need of mechanical ventilation greater than 24 hours post-surgery. The study was approved by research ethics committee of the first affiliated hospital, college of medicine, Zhejiang university (No. 876) and written informed consent was obtained from all patients. All procedures performed in this study involving human participants were in accordance with the Declaration of Helsinki (as revised in 2013).

\section{Intervention}

The intervention group received a daily post-operative physiotherapy intervention, including individually adapted mobilization and ambulation (the patients were instructed to gradually increase activity until ambulation was achieved with a walker whether or not, based on their requires) and breathing exercises (5 deep breaths each set for 3 sets, accomplished every hour), with explanations on coughing skills. At the first 2 days following surgery, all patients in the intervention group, received approximately the 
similar intensity of exercise (20-30 minutes every session), and then from the $3^{\text {rd }}$ day, the intervention program was made individualized based on the their conditions. The physiotherapy treatment, performed by a study-responsible physical therapist, was available from Monday to Friday. The control group did not receive any physiotherapy intervention or specific treatment during the in-hospital stay. The intervention would be discontinued, when their respiratory rate or heart rate increased of over $20 \%$ from baseline during the process.

\section{Outcomes}

The outcomes were the level of physical activity pre-operatively and on the 5th day after surgery. Baseline measurements of handgrip strength and the timed up and go test (TUG) were obtained on the day of admission. On the 5 th day after surgery, all the patients would be retest, as they were still in the hospital and able to complete the tests. And all these measurements were performed by a physical therapist who was blinded to the group allocation.

\section{Muscle strength}

It is simple and inexpensive to measure the strength of grip muscles. Low grip strength is considered a strong indicator of unfavorable outcomes such as longer hospitalization, greater functional limitations, lower health-related quality of life, and death $(13,14)$. Accurate measurement of grip strength requires using a handheld dynamometer under well-defined test situations, for which was taken from appropriate reference populations (15). The handgrip strength is moderately correlated with strength in other body parts, therefore, it is considered as a reliable alternative for more complicated measurements of extremity strength. Due to its ease of use, grip strength is recommended for routine use, especially in some communities and clinical settings $(13,14,16-18)$.

To obtain an optimal result, patients were required to grip the apparatus and then squeeze it 3 times as hard as possible, with at least $2 \mathrm{~min}$ breaks between attempts. In the study, patients' mean and peak measurements were recorded in kilograms. The patients were required to use their dominant hand. It is impossible to test handgrip strength if the hand is disabled (e.g., with stroke or advanced arthritis), so in such cases, lower limb strength can be measured using isometric torque methods (19-24).

\section{Physical performance}

Physical performance has been considered an objective measurement for total body function, which is associated with locomotion (25). It is a multidimensional measurement that includes functions of the musculoskeletal system and the nervous system, and also involves balance (26). Physical performance can be assessed by gait speed, the TUG test, and other measurements. Certain other measures may not be possible to obtain when a patient's performance is impaired by dementia, a balance impairment, or gait disorders.

To assess physical performance, the TUG test was used in this study. The test was performed on a 3 meter walkway. The patients were prompted to rise from sitting in a standard armchair, walk ahead for 3 meters, and then turn back to the chair, sit down. They were suggested to walk as quickly as they could, and they can walk with or without a walking aid, if needed. The result was recorded in seconds.

\section{Statistical methods}

For patient characteristics, continuous data are summarized as the mean \pm standard deviation (SD), and categorical data are presented as numbers and percentages. Differences between the groups were tested using the Student's $t$-test, while the differences of categorical variables were tested using the $\chi^{2}$ test. For all analyses, a $\mathrm{P}$ value $<0.05$ was considered statistically significant. All the analyses were performed using IBM Statistical Package Program for the Social Sciences 24 (IBM Corporation, Armonk, New York, USA).

\section{Results}

During the period from 2018 to 2019, a total of 34 patients at our institution were included in this study. One patient from the control group was excluded due to refusing surgery. Ultimately, the study consisted of 33 patients (intervention group $\mathrm{n}=17$, and control group $\mathrm{n}=16$ ) (Figure 1). The mean ages in the intervention and control groups were 59 (11.4) and 60 (14.4) years, respectively. Both in these two groups, 7 among participations were men (41\% in intervention group, $43 \%$ in control group). There were no statistically obvious differences in their demographic characteristics between the 2 groups $(\mathrm{P}>0.05)$. These data are presented in Table 1.

At baseline, there were no significant differences between these 2 groups in age, gender, body mass index, handgrip 


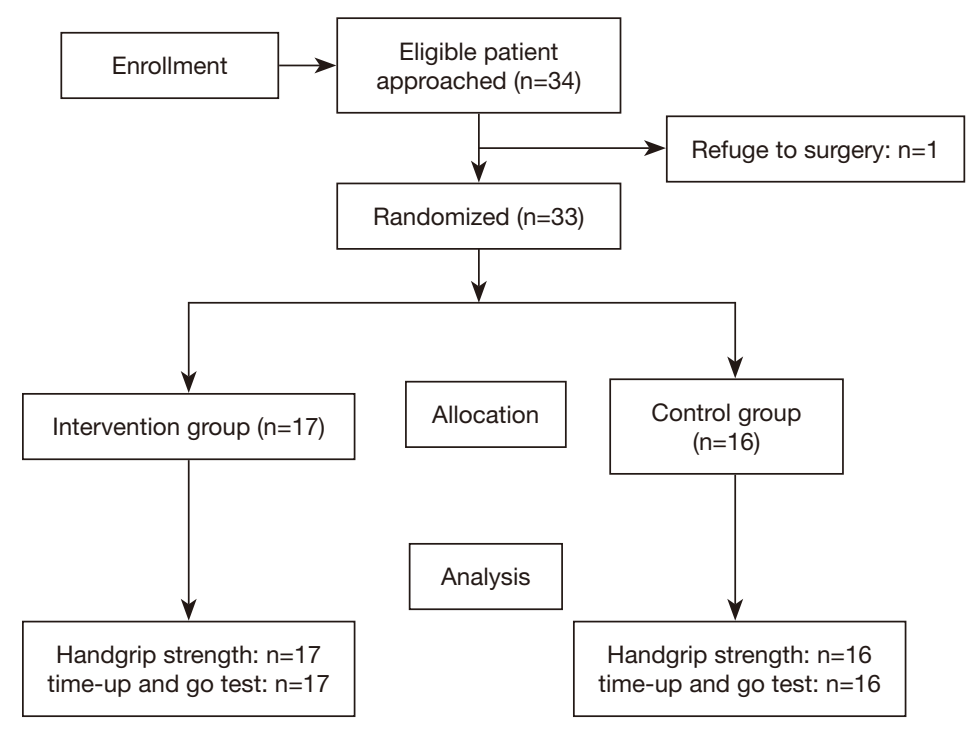

Figure 1 Study flow chart.

Table 1 Baseline characteristics of the study participants

\begin{tabular}{lccc}
\hline Characteristics & Treatment group $(\mathrm{n}=17)$ & Control group $(\mathrm{n}=16)$ & $\mathrm{P}$ value \\
\hline Age $($ years $)$ & $58.94 \pm 11.38$ & $60.19 \pm 14.39$ & 0.78 \\
Sex, male & $7(41.18 \%)$ & $7(43.75 \%)$ & 0.58 \\
Height $(\mathrm{m})$ & $1.61 \pm 0.05$ & $1.62 \pm 0.06$ & 0.81 \\
Weight $(\mathrm{kg})$ & $58.86 \pm 12.35$ & $60.91 \pm 9.36$ & 0.60 \\
BMI $\left(\mathrm{kg} / \mathrm{m}^{2}\right)$ & $22.62 \pm 4.02$ & $23.27 \pm 2.70$ & 0.59 \\
\hline
\end{tabular}

Data are demonstrated as mean $\pm \mathrm{SD}$, or number $(\mathrm{n})$ of participations. The $\mathrm{P}$ value makes reference to the difference between these two groups. BMI, body mass index; HSG, hand strength; TUG, timed up and go.

Table 2 Pre- and post-operative data for handgrip strength (HSG) and the timed up and go (TUG) test

\begin{tabular}{|c|c|c|c|c|c|c|c|}
\hline Variable & \multicolumn{3}{|c|}{ Treatment group $(n=17)$} & \multicolumn{3}{|c|}{ Control group $(n=16)$} & $P$ value \\
\hline HSG (kg) & $17.79 \pm 6.39$ & $20.58 \pm 7.17$ & $<0.001$ & $17.72 \pm 5.25$ & $17.43 \pm 6.73$ & 0.22 & $<0.001$ \\
\hline TUG (s) & $6.12 \pm 2.10$ & $6.32 \pm 2.73$ & 0.65 & $6.16 \pm 1.55$ & $6.46 \pm 1.71$ & 0.02 & 0.61 \\
\hline
\end{tabular}

Data are presented as mean \pm SD. The $\mathrm{P}$ value refers to the difference between the groups. BMI, body mass index; HSG, hand strength;

TUG, timed up and go.

strength, or physical performance parts (TUG test, Table 1). All these data were acquired before the patients received physiotherapy.

As for patients' follow-up tests on the 5 th post-operative day, there had significant differences between intervention group and control group in terms of handgrip strength and physical performance (TUG test, Table 2). There were some improvements in handgrip strength (from 17.79 to 20.58) and physical performance (from 6.12 to 6.32) in the treatment group, while handgrip strength (from 17.72 to 17.43 ) and muscle performance (from 6.16 to 6.46 ) markedly decreased in the control group. Among these data, only the data for handgrip strength between the 2 groups on day 5 after surgery were statistically significant. 


\section{Discussion}

This study demonstrated that patients who underwent physical therapy during the first 5 post-operative days after cardiac valve surgery had better physical strength and performance during their hospitalization than patients who did not receive physical therapy. Overall, patients in the treatment group achieved significantly greater strength and performance than the patients in the control group. Early post-operative mobilization is associated with multiple benefits, including improved pulmonary function, muscle strength, and physical performance. A system review of 15 randomized clinical trials on early mobilization in ICUs, discovered that early mobilization is executable, reliable and beneficial to patients' functional capacity (27).

In our study, the strength of handgrip muscles was obviously higher in the treatment group than in the control group. After 5 days of physiotherapy intervention, the increase of muscle strength in the intervention group was much quicker comparing to the other group. da Silva et al. (28) showed that handgrip strength appeared to be related to mechanical ventilation time, length of stay in the intensive care unite, and length of hospitalization in patients undergoing cardiac surgery. It has been considered that handgrip strength is an effective functional ability test to evaluating muscle strength not only in the general population, but also hospitalized and non-hospitalized ill persons $(29,30)$. This test has also been studied in the clinical environment, which has been regarded as a diagnostic tool to assess the risk of malnutrition and mortality (31).

In our study, muscle performance as measured by the TUG test did improve in the treatment group compared to the control group, however, there was no significant difference between these two groups. A deficiency of difference in physical performance between groups has been demonstrated in previous studies, supporting our current results. The intensity of the supervised post-operative walking along the corridor in hospital might too low to have an active effect on patient's functional capacity, as measured by the TUG in our study. Also, the 5 days of intervention might be not enough to show a positive result on the TUG. Studies have found that exercise training was beneficial to patient's exercise capacity in short-term improvements. They also found exercise in cardiac rehabilitation program might have an influence on the occurrence of poor events $(7,32)$. There was no any difference between intervention group and control group in any outcome measures in
Patman' study (33). However, many lines of evidence support the effect of early mobilization on functional performance. More studies are needed to clarify the effect of in-hospital physical activity after cardiac valve surgery.

The patients in our study were able to discharge, even though they were in a low physical activity level comparing to general population. That made a need for outpatient physical therapy. Doing cardiac rehabilitation at home after discharge could potentially increase patient's exercise capacity (14). Although the potential benefits of cardiac rehabilitation program (5), referring patients to participate in this program is rare in China. The most significant role of physical therapist during hospitalization is to inform patients about the benefits of cardiac rehabilitation. Although early physical activity after heart valve surgery is not known whether it matters, but our results suggest that having a good start is beneficial. Our study also show that physical therapists can play a positive role in helping patients be active as soon as possible during hospitalization.

\section{Limitations}

First, the sample size in our study was not large enough. Another limitation was that this study was not a doubleblind control trail. However, the character of physical therapy treatment makes it incapable to organize a doubleblind study. In order to diminish the potential bias, the assessments would be performed by a physical therapist who needed to be blinded to group allocation. Third limitation was lacking of a long-term follow-up. As physiotherapy is not well-accepted by some people in China, it is hard to carry out a follow-up intervention program after patients are discharged.

\section{Conclusions}

Patients who received 5 days of in-hospital physiotherapy showed improved levels of physical activity after cardiac valve surgery, comparing to the control group. While there has not been found any significant differences on the TUG test. The clinical value of increasing physical activity level during the early post-operative time requires a further study.

\section{Acknowledgments}

Funding: This work was supported by the Natural Science Foundation of Zhejiang Province (LGF18H170002). 


\section{Footnote}

Reporting Checklist: The authors have completed the CONSORT reporting checklist. Available at http://dx.doi. org/10.21037/apm-20-2259

Data Sharing Statement: Available at http://dx.doi. org/10.21037/apm-20-2259

Conflicts of Interest: All authors have completed the ICMJE uniform disclosure form (available at http://dx.doi. org/10.21037/apm-20-2259). Each author reports grants from the Natural Science Foundation of Zhejiang Province, during the conduct of the study. The authors have no other conflicts of interest to declare.

Ethical Statement: The authors are accountable for all aspects of the work in ensuring that questions related to the accuracy or integrity of any part of the work are appropriately investigated and resolved. The study was approved by research ethics committee of the first affiliated hospital, college of medicine, Zhejiang university (No. 876) and written informed consent was obtained from all patients. All procedures performed in this study involving human participants were in accordance with the Declaration of Helsinki (as revised in 2013).

Open Access Statement: This is an Open Access article distributed in accordance with the Creative Commons Attribution-NonCommercial-NoDerivs 4.0 International License (CC BY-NC-ND 4.0), which permits the noncommercial replication and distribution of the article with the strict proviso that no changes or edits are made and the original work is properly cited (including links to both the formal publication through the relevant DOI and the license). See: https://creativecommons.org/licenses/by-nc-nd/4.0/.

\section{References}

1. Ben-Dor I, Pichard AD, Gonzalez MA, et al. Correlates and causes of death in patients with severe symptomatic aortic stenosis who are not eligible to participate in a clinical trial of transcatheter aortic valve implantation. Circulation 2010;122:S37-42.

2. Vahanian A, Alfieri O, Andreotti F, et al. Guidelines on the management of valvular heart disease: the Task Force on the Management of Valvular Heart Disease of the European Society of Cardiology (ESC) and the European
Association for Cardio-Thoracic Surgery (EACTS). Eur Heart J 2012;33:2451-96.

3. Karlsson AK, Mattson B, Johansson M, et al. Well- being in patients and relatives after open-heart surgery from the perspective of health care professionals. J Clin Nurs 2010;19:840-6.

4. Hansen L, Winkel S, Kuhr J, et al. Factors influencing survival and postoperative quality of life after mitral valve reconstruction. Eur J Cardiothorac Surg 2010;37:635-44.

5. Fredericks S, Lapum J, Lo J. Anxiety, depression, and self-management: a systematic review. Clin Nurs Res 2012;21:411-30.

6. Whalley B, Rees K, Davies P, et al. Psychological interventions for coronary heart disease. Cochrane Database Syst Rev 2011;(8):CD002902.

7. Soares GMT, Ferreira DCS, Gonçalves MCP, et al. Prevalence of major postoperative complications in cardiac surgery. Rev Bras Cardiol 2011;24:139-46.

8. Freeman R, Maley K. Mobilization of intensive care cardiac surgery patients on mechanical circulatory support. Crit Care Nurs Q 2013;36:73-88.

9. Bourdin G, Barbier J, Burle JF, et al. The feasibility of early physical activity in intensive care unit patients: a prospective observational one-center study. Respir Care 2010;55:400-7.

10. Barbosa P, Santos FV, Neufejd PM, et al. Effects of early mobilization on cardiovascular and autonomic behavior in postoperative myocardial revascularization. ComSci Saúde 2010;9:111-7.

11. García-Delgado M, Navarrete-Sánchez I, Colmenero $M$. Preventing and managing perioperative pulmonary complications following cardiac surgery. Curr Opin Anaesthesiol 2014;27:146-52.

12. Adler J, Malone D. Early mobilization in the intensive care unit: a systematic review. Cardiopulm Phys Ther J 2012;23:5-13.

13. Ibrahim K, May C, Patel HP, et al. A feasibility study of implementing grip strength measurement into routine hospital practice (GRImP): study protocol. Pilot Feasibility Stud 2016;2:27.

14. Leong DP, Teo KK, Rangarajan S, et al. Prognostic value of grip strength: findings from the Prospective Urban Rural Epidemiology (PURE) study. Lancet 2015;386:266-73.

15. Roberts HC, Denison HJ, Martin HJ, et al. A review of the measurement of grip strength in clinical and epidemiological studies: towards a standardised approach. Age Ageing 2011;40:423-9. 
16. Rossi AP, Fantin F, Micciolo R, et al. Identifying sarcopenia in acute care setting patients. J Am Med Dir Assoc 2014;15:303.e7-12.

17. Steiber N. Strong or weak handgrip? Normative reference values for the German population across the life course stratified by sex, age, and body height. PLoS One 2016;11:e0163917.

18. Beaudart C, McCloskey E, Bruyere O, et al. Sarcopenia in daily practice: assessment and management. BMC Geriatr 2016;16:170.

19. Francis P, Toomey C, Mc Cormack W, et al. Measurement of maximal isometric torque and muscle quality of the knee extensors and flexors in healthy 50- to 70-year-old women. Clin Physiol Funct Imaging 2017;37:448-55.

20. McGregor RA, Cameron-Smith D, Poppitt SD. It is not just muscle mass: a review of muscle quality, composition and metabolism during ageing as determinants of muscle function and mobility in later life. Longev Healthspan 2014;3:9.

21. Maden-Wilkinson TM, Degens H, Jones DA et al. Comparison of MRI and DXA to measure muscle size and age-related atrophy in thigh muscles. J Musculoskelet Neuronal Interact 2013;13:320-8.

22. Cooper C, Fielding R, Visser M, et al. Tools in the assessment of sarcopenia. Calcif Tissue Int 2013;93:201-10.

23. Cawthon PM, Peters KW, Shardell MD, et al. Cutpoints for low appendicular lean mass that identify older adults with clinically significant weakness. J Gerontol A Biol Sci Med Sci 2014;69:567-75.

24. Tosato M, Marzetti E, Cesari M, et al. Measurement of muscle mass in sarcopenia: from imaging to biochemical markers. Aging Clin Exp Res 2017;29:19-27.

25. Landi F, Onder G, Russo A, et al. Calf circumference,

Cite this article as: Chen J, Zhang T, Bao W, Zhao G, Chen Z. The effect of in-hospital physiotherapy on handgrip strength and physical activity levels after cardiac valve surgery: a randomized controlled trial. Ann Palliat Med 2021;10(2):2217-2223. doi: 10.21037/apm-20-2259 frailty and physical performance among older adults living in the community. Clin Nutr 2014;33:539-44.

26. Beaudart C, Rolland Y, Cruz-Jentoft AJ, et al. Assessment of Muscle Function and Physical Performance in Daily Clinical Practice: A position paper endorsed by the European Society for Clinical and Economic Aspects of Osteoporosis, Osteoarthritis and Musculoskeletal Diseases (ESCEO). Calcif Tissue Int 2019;105:1-14.

27. Adler J, Malone D. Early mobilization in the intensive care unit: a systematic review. Cardiopulm Phys Ther J 2012;23:5-13

28. da Silva TK, Perry IDS, Brauner JS, et al. Performance evaluation of phase angle and handgrip strength in patients undergoing cardiac surgery: Prospective cohort study. Aust Crit Care 2018;31:284-90.

29. Noori N, Kovesdy CP, Bross R, et al. Novel equations to estimate lean body mass in maintenance hemodialysis patients. Am J Kidney Dis 2011;57:130-9.

30. Leal VO, Mafra D, Fouque D, et al. Use of handgrip strength in the assessment of the muscle function of chronic kidney disease patients on dialysis: a systematic review. Nephrol Dial Transplant 2011;26:1354-60.

31. Ling CH, Taekema D, de Craen AJ, et al. Hand-grip strength and mortality in the oldest old population: the Leiden 85-plus study. CMAJ 2010;182:429-35.

32. Sibilitz KL, Berg SK, Tang LH, et al. Exercise-based cardiac rehabilitation for adults after heart valve surgery. Cochrane Database Syst Rev 2016;3:CD010876.

33. Patman S, Sanderson D, Blackmore M. Physiotherapy following cardiac surgery: is it necessary during the intubation period? Aust J Physiother 2001;47:7-16.

(English Language Editor: C. Betlazar-Maseh) 